\title{
COPRIME FACTORIZATION WITH $J$ ALL-PASS DENOMINATOR: THE NONCANONICAL CASE
}

\author{
C. $\operatorname{Oară}^{\star}$, A. Varga ${ }^{\star \star}$ \\ ^Faculty of Automatic Control and Computers \\ University Polytechnica Bucharest \\ Austrului 34, RO-73115, Bucharest, Romania \\ Fax: +(40) 13234 234; Email: oara@popov.riccati.pub.ro \\ ${ }^{\star \star}$ German Aerospace Center, DLR - Oberpfaffenhofen \\ Institute of Robotics and System Dynamics \\ D-82234 Wessling, Germany. \\ Fax: +(49)-8153-28-1441, E-mail: andras.varga@dlr.de
}

Keywords: linear systems; coprime factorizations; $J$ all-pass; descriptor realizations; numerical algorithms.

\begin{abstract}
Given an arbitrary rational matrix $G$, we are interested to construct the class of coprime factorizations of $G$ with $J$-all pass denominators of McMillan degree as small as possible. Recently, we have given necessary and sufficient solvability conditions and a construction of the class of solutions in the canonical case in which the denominator has McMillan degree equal to the number of unstable poles of $G$. In this paper we extend the theory of coprime factorizations with minimal degree denominator to the noncanonical case.
\end{abstract}

\section{Introduction and Preliminaries}

Let $G$ be an arbitrary rational matrix (possible improper) and let $\Gamma_{g}$ be a given domain of the closed complex plane. A left coprime factorization (LCF) over $\Gamma_{g}$ of $G$ is a representation of the form $G=M^{-1} N$, with $N$ and $M$ rational matrices having poles only in $\Gamma_{g}$ and satisfying $M U+N V=I$ for certain rational matrices $U$ and $V$ with all poles in $\Gamma_{g}$.

In this paper we are interested in LCFs with denominators $M$ of smallest possible McMillan degree (which we call minimal degree factorizations). In addition, we require for the denominator to have a certain symmetry, i.e., to be $J$-all pass either with respect to the imaginary axis or to the unit circle.

Apparently, the theory of minimal degree coprime factorizations (with or without symmetry) has not been previously considered as such in the literature, although it brings important numerical advantages and it is crux to solving various problems encountered in the theory of linear systems and networks $[15,6]$, to canonical and noncanonical spectral factorizations of unstable rational matrices $[2,6]$, to several conjugation based approaches to nonstandard $H_{\infty}$ control problems $[16,17]$. The only noticeable exception is [1], where coprime factorizations are addressed with another requirement of minimality, namely the sum of the McMillan degrees of the denominator and nominator to be as small as possible.

Recently [8], we have started to study coprime factorization with minimal degree denominators. We have shown that the minimal degree of a LCF over $\Gamma_{g}$ (without any requirement for symmetry) is $n_{b}$, where $n_{b}$ is the number of poles of $G$ in $\Gamma_{g}$, and we have given a description in terms of realizations of all factors solving the minimal degree LCF over $\Gamma_{g}$. We developed in [8] also a theory of minimal degree LCFs with the additional requirement that the denominator has a certain symmetry (with respect to the imaginary axis or the unit circle). In [8] we have studied only LCFs with $J$ all-pass denominator of degree $n_{b}$ - the so-called canonical case. For the canonical case we have given necessary and sufficient solvability conditions and, when solutions exist, we have given a parameterized description of the factors in terms of associated realizations.

This paper is a continuation of [8]. Here we study the more technical case in which such canonical solutions do not exist. Precisely, we discuss the solution of noncanonical LCFs with $J$ all-pass denominators of McMillan degree as small as possible $\left(>n_{b}\right)$. The computation of factors in the canonical case relies on solving a generalized Lyapunov equation of order $n_{b}$. In the noncanonical case, the rank $r$ of the solution of the same Lyapunov equation allows to determine the minimal degree of the factorization as $2 n_{b}-r$. The noncanonical factors can then be constructed by embedding the solution of this Lyapunov equation of order $n_{b}$ into a $2 n_{b}-r$ order Lyapunov equation with 
nonsingular solution. The noncanonical case has important connections with the noncanonical Wiener-Hopf and $J$ spectral factorizations as discussed in [2] for proper and invertible rational matrices.

\subsection{Basic notation and definitions}

For a matrix $A$ we denote by $A^{*}$ its conjugate transpose and if $A$ is invertible by $A^{-*}$ its conjugated transpose inverse, respectively. By $\mathbb{C}, \mathbb{C}^{-}, \mathbb{C}^{+}, \mathbb{C}^{0}$, and $\mathbb{R}$ we denote the complex plane, the open left half plane, the open right half plane, the imaginary axis, and the real axis, respectively, and let $\overline{\mathbb{C}}:=\mathbb{C} \cup\{\infty\}$ be the closed complex plane, and $\overline{\mathbb{C}}^{-}:=\mathbb{C}^{-} \cup \mathbb{C}^{0} \cup\{\infty\}, \overline{\mathbb{C}}^{+}:=\mathbb{C}^{+} \cup \mathbb{C}^{0} \cup\{\infty\}$. Here "overbar" denotes closure. By $\mathbb{D}$ we denote the open unit disk and $\mathbb{D}^{c}=\overline{\mathbb{C}} \backslash \overline{\mathbb{D}}$ stands for the exterior of the closed unit disk, containing the infinity. By $\mathbb{F}$ we denote either $\mathbb{R}$ or $\mathbb{C}$.

Consider the disjoint partition of $\overline{\mathbb{C}}$ into a "good" region $\Gamma_{g}$ and a "bad" region $\Gamma_{b}$

$$
\overline{\mathbb{C}}=\Gamma_{g} \cup \Gamma_{b}
$$

A frequent interpretation of $\Gamma_{g}$ in system theory is related to the standard stability concept, that is, for linear continuous-time systems $\Gamma_{g}=\mathbb{C}^{-}$, while for linear discrete-time systems $\Gamma_{g}=\mathbb{D}^{-}$(or, sometimes, their closures).

We denote with $G^{\sim}$ the adjoint of $G$, where $G^{\sim}(s)=$ $G^{*}(-\bar{s})$ in continuous-time and $G^{\sim}(z)=G^{*}(1 / \bar{z})$ in discrete-time. In particular, if $G$ has real coefficients we have $G^{\sim}(s)=G^{T}(-s)$ and $G^{\sim}(z)=G^{T}(1 / z)$, respectively. Let $J$ be a signature matrix, i.e., a matrix satisfying $J=J^{*}=J^{-1}$. We say that the rational matrix $G$ is $J$ all-pass with respect to the imaginary axis if $G^{\sim} J G=J$, where $G^{\sim}$ denotes adjoint in continuous-time. Accordingly, we say that $G$ is $J$ all-pass with respect to the unit circle if $G^{\sim} J G=J$, where $G^{\sim}$ denotes now adjoint in discrete-time.

By definition, the McMillan degree of $G$ - denoted $\delta(G)$ - is the sum of the orders of all its poles (finite and infinite). Once a partition (1) is fixed, we have $\delta(G)=n_{b}+n_{g}$, where $n_{g}$ denotes the number of "good" poles in $\Gamma_{g}$ and $n_{b}$ denotes the number of "bad" zeros in $\Gamma_{b}$ (counting multiplicities).

\subsection{Descriptor realizations of rational matrices}

It is well known (see for example $[14,13])$ that any $p \times m$ rational matrix $G(\lambda)$ with coefficients in $\mathbb{F}$ (even improper or polynomial) has a descriptor realization of the form

$$
G(\lambda)=\left[\begin{array}{c|c}
A-\lambda E & B \\
\hline C & D
\end{array}\right]:=C(\lambda E-A)^{-1} B+D,
$$

where $A, E \in \mathbb{F}^{n \times n}, B \in \mathbb{F}^{n \times m}, C \in \mathbb{F}^{p \times n}, D \in \mathbb{F}^{p \times m}$, and the so called pole pencil $A-\lambda E$ is regular, i.e., it is square and $\operatorname{det}(A-\lambda E) \not \equiv 0$. The dimension $n$ of the square matrices $A$ and $E$ is called the order of the realization (2). We use $\Lambda(A-\lambda E)$ to denote the union of generalized eigenvalues of the regular pencil $A-\lambda E$ (finite and infinite, multiplicities counting). The descriptor realization (2) of $G$ is called minimal if its order is as small as possible among all realizations of this kind.

The principal drawback of realizations of the form (2) is that their minimal possible order is greater than the McMillan degree of $G$, unless $G$ is proper, and this brings important technical difficulties in factorization problems in which the McMillan degree plays a paramount role. A remedy to this is to use a generalization of (2) in which either the " $B$ " or the " $C$ " matrix is replaced by a matrix pencil, as explained further. Any rational matrix $G$ has a realization

$$
\begin{aligned}
G(\lambda) & =\left[\begin{array}{c|c}
A-\lambda E & B-\lambda F \\
\hline C & D
\end{array}\right] \\
& :=C(\lambda E-A)^{-1}(B-\lambda F)+D,
\end{aligned}
$$

and for any fixed $\alpha, \beta \in \mathbb{F}$, not both zero, there exists a realization

$$
\begin{aligned}
G(\lambda) & =\left[\begin{array}{c|c}
A-\lambda E & B(\alpha-\lambda \beta) \\
\hline C & D
\end{array}\right] \\
& :=C(\lambda E-A)^{-1} B(\alpha-\lambda \beta)+D,
\end{aligned}
$$

where $A, E \in \mathbb{F}^{n \times n}, B, F \in \mathbb{F}^{n \times m}, C \in \mathbb{F}^{p \times n}, D \in$ $\mathbb{F}^{p \times m}$, and the pole pencil $A-\lambda E$ is regular. A realization (4) will be called centered (at $\frac{\alpha}{\beta}$; if $\beta=0$ we interpret $\frac{\alpha}{\beta}$ as $\infty)$. For details about these type of realisations we refer to [7].

As a methodological question, throughout the paper we assume that the rational matrix $G$ to start with is given by a minimal descriptor realization (4) as this type of realization is most frequently used in the literature to represent arbitrary (possibly improper) rational matrices, while the solutions $N$ and $M$ to the LCF $G=M^{-1} N$ will be given directly by minimal realizations (3) and (4) of order equal to their respective McMillan degree. Furthermore, once a partition (1) is fixed, we assume that $G$ is given by a separated realization with respect to (1), namely

$$
G(\lambda)=\left[\begin{array}{cc|c}
A_{b}-\lambda E_{b} & A_{b g}-\lambda E_{b g} & B_{b} \\
O & A_{g}-\lambda E_{g} & B_{g} \\
\hline C_{b} & C_{g} & D
\end{array}\right]
$$

where the $n_{b} \times n_{b}$ pencil $A_{b}-\lambda E_{b}$ contains the $n_{b}$ poles of $G(\lambda)$ in $\Gamma_{b}$ and

$$
\operatorname{rank}\left[\begin{array}{cc}
E_{b} & E_{b g}
\end{array}\right]=n_{b},
$$

that is, all infinite nondynamic modes are included in $A_{g}-\lambda E_{g}$. Starting with an arbitrary minimal realization (2) it is always possible to arrive to a separated realisation (6) by performing solely orthogonal transformations. Furthermore, if the realization to start with has real coefficients, and $\Gamma_{g}$ is symmetric, we can always determine a separated realization with real coefficients as well. 


\section{LCF with $J$ all-pass denomina- tor with respect to the imagi- nary axis}

In this section we give the solution to the minimal degree $\mathrm{LCF}$ with the requirement on the denominator to have a certain symmetry, namely we consider here the case in which the denominator is $J$ all-pass with respect to the imaginary axis. To reflect this symmetry accordingly, we take throughout this section the partition (1) defined by

$$
\Gamma_{b}:=\mathbb{C}^{+}\left(\text {or } \Gamma_{b}:=\mathbb{C}^{-}\right) \quad \Gamma_{g}:=\overline{\mathbb{C}} \backslash \Gamma_{b} .
$$

However, due to the additional requirement on the denominator to be $J$ all-pass it is not always possible to solve the LCF over $\Gamma_{g}$ with minimal degree $n_{b}$. When this is possible, we call the factorization canonical, otherwise we call it noncanonical. The noncanonical case is considerably more intricate than the canonical case. The idea in the noncanonical case is to introduce additional poles and zeros in $M(s)$ such that it could simultaneously be $J$ all-pass and solve the LCF problem for $G(s)$. At the same time, we want to keep the McMillan degree of $M(s)$ as small as possible. It turns out that the additional poles/zeros can be taken only on the imaginary axis (including infinity) since the pole-zero symmetry featured by a $J$ all-pass factor implies with necessity that all additional poles will be reflected into symmetric additional zeros that will be also zeros of the compound matrix $[N(s) M(s)]$.

Before stating our main result we construct a particular separated realization of $G$ which facilitates the subsequent developments. Let $G(s)$ be an arbitrary rational matrix given by a minimal realization (5) separated with respect to $\Gamma_{g} \cup \Gamma_{b}$ and satisfying (6), and let $n_{b}$ be the number of poles of $G$ in $\Gamma_{b}$. Then the generalized Lyapunov equation

$$
A_{b}^{*} X E_{b}+E_{b}^{*} X A_{b}-C_{b}^{*} J C_{b}=0
$$

has a unique Hermitic solution $X$, and let $r:=\operatorname{rank}(X)$. Let

$$
U^{*} X U=\left[\begin{array}{cc}
\Sigma_{r} & O \\
O & O
\end{array}\right]
$$

be a spectral decomposition of $X$ where $\Sigma_{r}$ is diagonal and nonsingular and $U$ is unitary. Further, let

$$
U^{*} E_{b} Q^{*}=\left[\begin{array}{cc}
E_{11} & O \\
E_{21} & E_{22}
\end{array}\right]
$$

be a RQ decomposition of $U^{*} E_{b}$, where $Q$ is unitary, $E_{11}$ and $E_{22}$ are lower triangular, square and invertible, and the partition in (10) corresponds to the partition in (9). With (9) and (10) it is easy to see that

$$
G(s)=\left[\begin{array}{cc|c}
U^{*}\left(A_{b}-s E_{b}\right) Q^{*} & U^{*}\left(A_{b g}-s E_{b g}\right) & U^{*} B_{b} \\
O & A_{g}-s E_{g} & B_{g} \\
\hline C_{b} Q^{*} & C_{g} & D
\end{array}\right]
$$

is another realization of $G$ separated with respect to $\Gamma_{g} \cup$ $\Gamma_{b}$ which we call balanced with respect to the Lyapunov equation (8). Thus we may assume from the beginning that for the separated realization $(5)$ we have $E_{b}$ lower triangular and the corresponding Lyapunov equation (8) has a diagonal solution. We are now ready for our main existence result.

Theorem 2.1 Given an arbitrary rational matrix $G(s)$ and a disjoint partition $\overline{\mathbb{C}}=\Gamma_{g} \cup \Gamma_{b}$ defined by (7), let $n_{b}$ be the number of poles of $G$ in $\Gamma_{b}$. Assume (5) is a minimal descriptor realization of $G$, separated with respect to the given partition, and satisfying the condition (6). Let $r:=\operatorname{rank}(X)$ where $X$ is the unique Hermitic solution to the Lyapunov equation

$$
A_{b}^{*} X E_{b}+E_{b}^{*} X A_{b}-C_{b}^{*} J C_{b}=0 .
$$

Then there exists a LCF with $J$ all-pass denominator and the minimal McMillan degree of such a LCF is

$$
2 n_{b}-r .
$$

Sketch of proof. Without restricting the generality, we may assume from the beginning that the realization (5) is balanced with respect to the Lyapunov equation (12). Further, as $E_{b}$ is invertible we could, as a first simplifying step in the whole proof, reduce the equation (12) to a standard Lyapunov equation with $E_{b}=I$. However, we prefer not to invert $E_{b}$ as far as possible as this will bring benefits in terms of the reliability of the associated numerical algorithms.

The proof is quite lengthy and we divide it in several steps: we show first that any solution $G=M^{-1} N$ to the LCF with $J$ all pass denominator satisfies

$$
\delta(M) \geq 2 n_{b}-r,
$$

and prove further that $M^{-1}$ has exactly $n_{b}$ poles in $\Gamma_{b}$ (which are the poles of $G$ in $\Gamma_{b}$ ) and the rest (additional) $\delta(M)-n_{b}$ poles are with necessity on $\overline{\mathbb{C}}^{0}$. Finally, we construct a solution of minimal degree $2 n_{b}-r$ with the additional $n_{b}-r$ poles placed arbitrary on $\overline{\mathbb{C}}^{0}$.

Proof of $\delta(M) \geq 2 n_{b}-r$. Let $G(s)=M^{-1}(s) N(s)$ be a solution to the LCF with $J$ all pass denominator. As $M$ cancels in the product $N=M G$ all poles of $G$ in $\Gamma_{b}$ and $M^{-1}$ is $J$ all-pass, we show that there exists a minimal realization of $M^{-1}$ of the form

$$
\begin{aligned}
M^{-1}(s) & =\left[\begin{array}{c|c}
A_{M}-s E_{M} & B_{M}(\alpha-s \beta) \\
\hline C_{M} & D_{M}
\end{array}\right] \\
& =\left[\begin{array}{cc|c}
A_{b}-s E_{b} & A_{b x}-s E_{b x} & B_{x 1}(\alpha-s \beta) \\
O & A_{x}-s E_{x} & B_{x 2}(\alpha-s \beta) \\
\hline C_{b} & C_{x} & D_{M}
\end{array}\right]
\end{aligned}
$$

and there exists a Hermitic invertible matrix $X_{M}$ such that

$$
A_{M}^{*} X_{M} E_{M}+E_{M}^{*} X_{M} A_{M}-C_{M}^{*} J C_{M}=0 .
$$


According to the partition of the right-hand side of (15), $X$ can be written in the partitioned form

$$
X_{M}=\left[\begin{array}{cc}
X & X_{12} \\
X_{12}^{*} & X_{22}
\end{array}\right]
$$

where it follows that $X$ is the unique solution to (12). Since $X_{M}$ is invertible and the rank of $X$ is $r$, it follows from (17) that rank $\left(X_{M}\right) \geq 2 n_{b}-r$ and thus (14) holds.

Location of the poles of $M^{-1}$. Let $G=M^{-1} N$ be a solution to the LCF with $J$ all pass denominator. From (15) we see that the union of poles of $M^{-1}(s)$ contains

$$
\Lambda\left(A_{b}-s E_{b}\right) \subset \mathbb{C}^{+}
$$

which are also the poles of $G$ in $\Gamma_{b}$. From the polezero symmetry of a $J$ all-pass rational matrix it follows that the poles of $M(s)$ are the conjugated of the poles of $M^{-1}(s)$. Since $M$ has all poles in $\Gamma_{g}$ we infer that the poles of $M^{-1}$ are in $\bar{\Gamma}_{b}:=\Gamma_{b} \cup \overline{\mathbb{C}}^{0}$ from where we have

$$
\Lambda\left(A_{x}-s E_{x}\right) \subset \bar{\Gamma}_{b} .
$$

We show further that

$$
\Lambda\left(A_{x}-s E_{x}\right) \subset \Gamma_{g},
$$

from where we shall conclude with (19) that

$$
\Lambda\left(A_{x}-s E_{x}\right) \subset \overline{\mathbb{C}}^{0}
$$

and thus $M^{-1}$ has exactly $n_{b}$ poles in $\Gamma_{b}$ and the additional $\delta(M)-n_{b}$ poles are on $\overline{\mathbb{C}}^{0}$. We prove in fact that the coprimeness of $N$ and $M$ over $\Gamma_{g}$ implies (20).

Construction of a minimal solution. We show now that the LCF with $J$ all-pass denominator has a solution $G=M^{-1} N$ such that

$$
\delta(M) \leq 2 n_{b}-r
$$

from where it will follow with (14) that the minimal degree $2 n_{b}-r$ can be achieved. To this end, we shall construct $M(s)$, define $N(s)=M(s) G(s)$, and prove that they both satisfy the required properties. Of course, $M$ should simultaneously be $J$ all-pass, and $M^{-1}$ should have the form (15), with the additional poles given by $\Lambda\left(A_{x}-s E_{x}\right) \subset \overline{\mathbb{C}}^{0}$.

For the sake of clarity we assume first that the additional poles/zeros $\Lambda\left(A_{x}-s E_{x}\right)$ are placed in finite locations on $\mathbb{C}^{0}$. The case with poles at infinity follows analogously although the formulas become more intricate.

Placing additional poles/zeros in finite locations on $\mathbb{C}^{0}$. In this case we can take for $M^{-1}$ a realization (15) with $\alpha=1, \beta=0$, i.e.,

$$
\begin{aligned}
M^{-1}(s) & =\left[\begin{array}{c|c}
A_{M}-s E_{M} & B_{M} \\
\hline C_{M} & D_{M}
\end{array}\right] \\
& =\left[\begin{array}{cc|c}
A_{b}-s E_{b} & A_{b x}-s E_{b x} & B_{x 1} \\
O & A_{x}-s E_{x} & B_{x 2} \\
\hline C_{b} & C_{x} & D_{M}
\end{array}\right] .
\end{aligned}
$$

The key idea of the construction is to embed the Lyapunov equation (12) into a larger Lyapunov equation (16) with an invertible solution $X_{M}$ as in (17) and where the matrices $A_{x}, E_{x}, A_{b x}, E_{b x}, B_{x 1}, B_{x 2}, C_{x}$ have to be determined. We can simply take

$$
E_{b x}=0, \quad E_{x}=I_{n_{b}-r}
$$

in case of placing additional poles/zeros at finite locations. We choose further

$$
X_{12}=\left[\begin{array}{c}
O \\
I_{n_{b}-r}
\end{array}\right], \quad X_{22}=O
$$

and get

$$
\begin{aligned}
& X_{M}=\left[\begin{array}{ccc}
\Sigma_{r} & O & O \\
O & O & I_{n_{b}-r} \\
O & I_{n_{b}-r} & O
\end{array}\right] \text {, } \\
& E_{M}=\left[\begin{array}{ccc}
E_{11} & O & O \\
E_{21} & E_{22} & O \\
O & O & I_{n_{b}-r}
\end{array}\right] \text {, } \\
& \begin{aligned}
A_{M} & =\left[\begin{array}{ccc}
A_{11} & A_{12} & A_{x 1} \\
A_{21} & A_{22} & A_{x 2} \\
O & O & A_{x}
\end{array}\right], \\
C_{M} & =\left[\begin{array}{ccc}
C_{1} & C_{2} & C_{x}
\end{array}\right],
\end{aligned}
\end{aligned}
$$

where we have taken into account that the realization (5) is balanced with respect to (12) and we have partitioned

$A_{b}=\left[\begin{array}{ll}A_{11} & A_{12} \\ A_{21} & A_{22}\end{array}\right], A_{b x}=\left[\begin{array}{l}A_{x 1} \\ A_{x 2}\end{array}\right], C_{b}=\left[\begin{array}{ll}C_{1} & C_{2}\end{array}\right]$,

conformably with (9). The matrices $A_{x 1}, A_{x 2}, A_{x}, C_{x}$ remain to be determined as to satisfy (16). We use (25) to write (16) component-wise as

$$
\begin{gathered}
A_{11}^{*} \Sigma_{r} E_{11}+E_{11}^{*} \Sigma_{r} A_{11}-C_{1}^{*} J C_{1}=0, \\
E_{11}^{*} \Sigma_{r} A_{12}-C_{1}^{*} J C_{2}=0, \\
C_{2}^{*} J C_{2}=0, \\
A_{21}^{*}+E_{11}^{*} \Sigma_{r} A_{x 1}+E_{21}^{*} A_{x}-C_{1}^{*} J C_{x}=0, \\
A_{22}^{*}+E_{22}^{*} A_{x}-C_{2}^{*} J C_{x}=0, \\
A_{x 2}^{*}+A_{x 2}-C_{x}^{*} J C_{x}=0,
\end{gathered}
$$

where the rest of equations are transpose conjugated versions of the ones above. Equations (26), (27) and (28) are automatically fulfilled due to (12). Before showing how the remaining equations can be satisfied, we prove that the pair $\left(C_{2}, A_{22}-s E_{22}\right)$ is observable. It follows from (30) that we can take the matrix $C_{x}$ such that the poles of $A_{x}=E_{22}^{-*}\left(-A_{22}^{*}+C_{2}^{*} J C_{x}\right)$ are on the imaginary axis by simply solving an eigenvalue assignment problem for the controllable pair

$$
\left(-E_{22}^{-*} A_{22}^{*}, E_{22}^{-*} C_{2}^{*} J\right) .
$$

Finally, we choose $A_{x 1}$ as the unique solution of (29) and $A_{x 2}$ as any solution to (31). With all these choices it results that (16) is satisfied. 
We take $D_{M}$ to be any $J$ unitary matrix and define

$$
B_{M}=\left[\begin{array}{c}
B_{x 1} \\
B_{x 2}
\end{array}\right]:=E_{M}^{-*} X_{M}^{-1} C_{M}^{*} D_{M}^{*} J
$$

We get that $M^{-1}$ is $J$ all pass. It follows that $M$ has all its poles in $\Gamma_{g}$, is $J$ all-pass, has McMillan degree less or equal to $2 n_{b}-r$, and a realization is given by

$$
\begin{gathered}
M(s)=\left[\begin{array}{c|c}
A_{M}-s E_{M}-B_{M} W C_{M} & -B_{M} W \\
\hline W C_{M} & W
\end{array}\right]= \\
{\left[\begin{array}{cc|c}
A_{b}-s E_{b}-B_{x 1} W C_{b} & A_{b x}-B_{x 1} W C_{x} & -B_{x 1} W \\
-B_{x 2} W C_{b} & A_{x}-s I-B_{x 2} W C_{x} & -B_{x 2} W \\
\hline W C_{b} & W C_{x} & W
\end{array}\right]}
\end{gathered}
$$

where $W=D_{M}^{-1}$. We define $N(s):=M(s) G(s)$ and get

$$
\begin{gathered}
N(s)=\left[\begin{array}{cc}
A_{b}-s E_{b}-B_{x 1} W C_{b} & A_{b x}-B_{x 1} W C_{x} \\
-B_{x 2} W C_{b} & A_{x}-s I-B_{x 2} C_{x} \\
O & O \\
\hline W C_{b} & W C_{x} \\
& \\
A_{b g}-B_{x 1} W C_{g}-s E_{b g} & B_{b}-B_{x 1} W D \\
-B_{x 2} W C_{g} & -B_{x 2} W D \\
A_{g}-s E_{g} & B_{g} \\
\hline W C_{g} & W D
\end{array}\right]
\end{gathered}
$$

We can check successively that $N$ has all poles in $\Gamma_{g}$, and that $N$ and $M$ are coprime over $\Gamma_{g}$. Thus $N$ and $M$ define a solution to the LCF with $J$ all-pass denominator, having degree less or equal to $2 n_{b}-r$. This together with (14) shows that the minimal degree $2 n_{b}-r$ is attained and concludes the proof.

Placing additional poles/zeros arbitrary on $\overline{\mathbb{C}^{0}}$. This case can be reduced by an appropriate transformation to the case of finite poles/zeros.

The following theorem gives a characterization of the class of all solutions to the minimal degree LCF with $J$ all-pass denominator.

Theorem 2.2 Assume the same hypotheses and notation as for Theorem 2.1. The class of all solutions to the minimal degree $L C F$ with $J$ all-pass denominator $G=M^{-1} N$, with $M$ proper, is given by (34) and (35), where $C_{x}$ and $A_{x}$ follow by solving the eigenvalue assignment problem for the pair (32) with $A_{x}=E_{22}^{-*}\left(-A_{22}^{*}+C_{2}^{*} J C_{x}\right) \subset \mathbb{C}^{0}$, $A_{x 2}$ is any solution to (31), $A_{x 1}$ is the unique solution to (29), $W$ is any $J$ unitary matrix, and $B_{x 1}$ and $B_{x 2}$ are given by (33).

The proof is omitted for brevity. The case in which $M$ is allowed to be improper can be treated analogously although the formulae are more intricate.

\section{LCF with $J$ all-pass denomina- tors with respect to the unit cir- cle}

In this section we sketch the discrete-time version of the results presented in the previous section. More precisely, we solve the minimal degree LCF problem with the additional requirement on the denominator to have another type of symmetry, namely to be $J$ all-pass with respect to the unit circle. Throughout this section we take the partition (1) defined by

$$
\Gamma_{b}:=\mathbb{D}^{c}\left(\text { or } \Gamma_{b}:=\mathbb{D}\right), \quad \Gamma_{g}:=\overline{\mathbb{C}} \backslash \Gamma_{b} .
$$

Similarly as for the symmetry discussed in the previous section, it is not always possible to solve the LCF over $\Gamma_{g}$ with minimal degree $n_{b}$. Again, when this is possible, we call the factorization canonical, otherwise we call it noncanonical.

Theorem 3.1 Let $G(z)$ be a rational matrix and let $n_{b}$ be the number of poles of $G(z)$ in $\Gamma_{b}$. Consider a minimal descriptor realization of $G(z)$ separated with respect to the partition $\Gamma_{g} \cup \Gamma_{b}$ in the form (5) and satisfying (6). Let $X$ be the unique hermitic solution to the Lyapunov equation

$$
E_{b}^{*} X E_{b}-A_{b}^{*} X A_{b}-C_{b}^{*} J C_{b}=0 .
$$

Then the LCF with $J$ all-pass denominator with respect to the unit circle has a solution of minimal degree

$$
2 n_{b}-\operatorname{rank}(X)
$$

Proof. The proof is much similar to the proof of Theorem 2.1. The key is to transform first equation (37) into the equation

$\left(E_{b}+A_{b}\right)^{*} X\left(E_{b}-A_{b}\right)+\left(E_{b}-A_{b}\right)^{*} X\left(E_{b}+A_{b}\right)-2 C_{b}^{*} J C_{b}=0$

where $E_{b}-A_{b}$ is invertible. Note that (39) is of the form (12) and we may use further a similar technique as in the proof of Theorem 2.1.

Similarly to Theorem 2.2, we can characterise the class of all solutions in the discrete-time case. The details are omitted.

\section{Conclusions}

We have extended the theory of coprime factorisations with minimal degree $J$ all pass denominator to the noncanonical case. The approach taken in this paper puts ground also for extensions to LCF with denominators satisfying other symmetries, as for example with respect to the real line, or with respect to a certain countour in the complex plane. The theory presented is a first step towards developing a comprehensive state-space theory of 
$J$ spectral factorizations, either canonical or noncanonical. The most important application of the results presented here is in the solution to the optimal (as oposed to suboptimal) $H^{\infty}$ control problem under the most relaxed poosible assumptions. These results will be presented in a forthcoming report.

\section{Acknowledgement}

This research has been made possible by a Fellowship of the Alexander von Humboldt Foundation, Bonn.

\section{References}

[1] Ball J.A., Kim J., Rodman L., Verma M., "Minimal degree coprime factorization of rational matrix functions", Linear Algebra and Its Applications, 186, 117-164, (1993).

[2] Bart H., Gohberg I., Kaashoek M. A., Constructive Methods of Wiener-Hopf Factorization. Birkhäuser, Basel, OT 21, (1986).

[3] Gantmacher F. R., The Theory of Matrices. Chelsea, New York, (1960).

[4] Gohberg I., Kaashoek M. A., Ran A.C.M, "Partial pole and zero displacement by cascade connection" SIAM J. Matrix Anal. Appl., 10(3), 316-325, (1989).

[5] Kimura H., Chain-Scattering Approach to $\mathrm{H}_{\infty}$ Control, Birkhäuser, Boston, (1996).

[6] Ionescu V., Oară C., Weiss M., Generalized Riccati Theory and Robust Control: A Popov Function Approach, John Wiley \& Sons, New York, (1999), ISBN 0-471-97147-2.

[7] Oară C., "Minimal Factorization of Rational Matrices: the General Case" to appear in Proceedings of MTNS'98, Padova, Italy, 6-10 July, (1998).

[8] Oară C., Varga A., Least Order Coprime Factorizations of Rational Matrices: The Canonical Case. Proceedings of 37th CDC, Tampa, Florida, December (1998).

[9] Rosenbrock H. H., State-Space and Multivariable Theory. Wiley, New York, (1970).

[10] Van Dooren P., "The generalized eigenstructure problem in linear systems theory", IEEE Trans. Autom. Control, 26, 111-129, (1981).

[11] Van Dooren P., "A generalized eigenvalue approach for solving Riccati equations", SIAM J. Sci. Stat. Comput., 2, 121-135, (1981).
[12] Van Dooren P., "Rational and polynomial matrix factorizations via recursive pole-zero cancellation", Lin. Alg. \& Appl., 137/138, 663-697, (1990).

[13] Verghese G., Lévy B., Kailath T., "A generalized state-space for singular systems" IEEE Trans. Autom. Control, 26, 811-831, (1981).

[14] Verghese G., Van Dooren P., Kailath T., "Properties of the system matrix of a generalized state-space system" Int. J. Control, 30, 235-243, (1979).

[15] Vidyasagar M., Control System Synthesis: A Factorization Approach, The MIT Press, Cambridge, MA, (1985).

[16] Xin X., Kimura H., " $\left(J, J^{\prime}\right)$ lossless factorization for descriptor systems", Lin. Alg. \& Appl., 205-206, 1289-1318, (1994).

[17] Xin X., Kimura H, "Singular $\left(J, J^{\prime}\right)$ lossless factorization for strictly proper functions" Int. J. Control, 59, 1383-1400, (1994). 